\title{
Transformación de los hábitos de práctica de actividad física y deporte dela población mayor de 65 años en Gipuzkoa The change in the sports and physical activities habits of over 65 year olds in the province of Gipuzkoa
}

\author{
*Juan Aldaz, Silvia Arribas, Lorena Gil de Montes e Izaskun Luis de Cos \\ *Escuela Universitaria de Magisterio de Donostia-San Sebastian (España)
}

\begin{abstract}
Resumen: El presente trabajo se deriva de una investigación más amplia llevada a cabo por el grupo de investigación IKERKI 05/30 reconocido por la UPV/EHU en la que se encuestó a una muestra representativa de la población guipuzcoana mayor de 18 años sobre sus hábitos de práctica de actividad física y deportiva. En el mismo se entrevisto a 288 sujetos mayores de 65 años con un nivel de error resultante de $\pm 5,9 \%$. A grandes rasgos, los hábitos de práctica de actividad física y deportiva (PAFYD) de la población guipuzcoana mayor de 65 años nos muestran cómo la población adulta, con el paso de los años, va tendiendo hacia una PAFYD menos institucionalizada, lo cual no quiere decir que se dé un abandono sino que nos hace pensar que la dinámica preformativa de los hábitos de PAFYD tiene que ver más con una transformación que con el abandono de los mismos. Este hecho nos hace pensar que se hace necesaria la reformulación de los modelos de institucionalización predominantes así como las políticas relacionadas con los hábitos de PAFYD de la población adulta, en general, y de los mayores de 65 años, en concreto.
\end{abstract}

Palabra clave: Hábitos de PAFYD, transformación, institucionalización, población mayor de sesenta y cinco años.

\begin{abstract}
The current study has its foundations in a more broad-ranging research initiative undertaken by the IKERI O5/30 research group (oficially recognised by the University of the Basque Country) which surveyed a representative sample of the over-18s population of Gipuzkoa in relation to their sports and physical activities habits. The cited research project interviewed 288 people of 65 and produced results within a $\pm 5,9 \%$ margin of error range. In very general terms the sports and physical activities habits of the cited over-65s generation (PAFYD) in the province of Gipuzkoa demonstrate that, with the passing of the years, the adult population becomes less reliant on state-sponsored options in terms of chosen dedication to sports and physical activities. This, by no means implies that this particular populous (the over 65s) abandons such activity but rather that there is a transformation in the type of activity that their age group participate in. This fact draws us to the conclusion that a re-think in terms of state promoted activities and models for the over-65s (PAFYD), in particular, and for the adult population, in general, is necessary.
\end{abstract}

Key words: Sport and physical activity Habits, transformation, institutionalization, Over 65s.

\section{Introducción}

Los seres humanos somos seres constructores y en construcción, es por ello que sea cual sea nuestra edad estamos en continuo movimiento a lo largo de toda nuestra vida. Se podría afirmar pues, que en el fondo del ideal Homo Faber, hay una característica «esencial» relativa a nuestro carácter de Homo Impiger, es decir, seres activos que en nuestro día a día necesitamos movernos, realizar actividad física para poder subsistir.

En este sentido, y haciendo nuestra la definición consensuada por el proyecto Europeo COMPASS y que se recoge en la Carta Europea del Deporte del Consejo Europeo (1992): «Se entenderá por $<<$ deporte $>>$ todo tipo de actividades físicas realizadas que, mediante una participación, organizada o de otro tipo, tengan por finalidad la expresión o la mejora de la condición física y psíquica, el desarrollo de las relaciones sociales o el logro de resultados en competiciones de todos los niveles». Nosotros al hacer referencia a este término tan amplio preferimos hablar de practica de actividad física y deporte, en adelante PAFYD, en los términos planteados en su tesis por Arribas (2004).

Tal y cómo señala el Doctor Campillo en su trabajo «El mono obeso» (2004), se podría afirmar que nuestro diseño genético es el mismo queel denuestros antepasados de hacemillones de años, obligados a estar casi en movimiento continuo para poder subsistir. Resumiendo, se podría afirmar que estamos diseñados biológicamente para movernos, para estar activos. No obstante, no todo movimiento, no toda actividad física, puede ser considerada deportiva.

Partiendo de esta premisa, el fenómeno deportivo contemporáneo, dada su enorme y creciente diversificación, se muestra como una realidad difícil de acotar a la hora de tratar de estudiar los hábitos de PAFYD, por lo que nos planteamos el tratar de comprenderlo como un sistema social

Fecha recepción: 15-01-10 - Fecha envío revisores: 15-01-10 - Fecha de aceptación: 15-01-10 Correspondencia: Juan Aldaz Plaza Oñati $N^{\circ} 3$,

Donostia 20018. Gipuzkoa

E-mail: juan.aldaz@ehu.es abierto (Puig y Heinemann, 1991) donde se van (re)generando nuevas prácticas y concepciones de la misma. Estos autores sugieren que ya no existe un único modelo explicativo que agrupe las características principales del deporte por lo que «Si se tienen en cuenta como dimensiones constituyentes la forma como se organizan las actividades deportivas, el modo como se legitiman, las motivaciones de los participantes y los impactos que producen, cabe sugerir cuatro modelos configuradores del deporte contemporáneo (...): competitivo, expresivo, instrumental y espectáculo» (Puig y Heinemann, 1991:126). Cabe decir que estos modelos han de ser entendidos, tal y como advierten los autores, como tipos ideales en el sentido descrito por Max Weber.

Dehecho, Puigy Heinemann(1991:125) destacan la diversificación como tendencia más relevante del «sistema deportivo contemporáneo». Así, insisten en el hecho de que «el deporte ha dejado de ser un sistema autónomo y se ha convertido en un sistema abierto con escasa identidad propia y estrechamente conectado a otros sistemas tales como el económico, el educativo, el político...» que podría ilustrarse, tal y como lo hacen los autores, en el esquema que sigue:

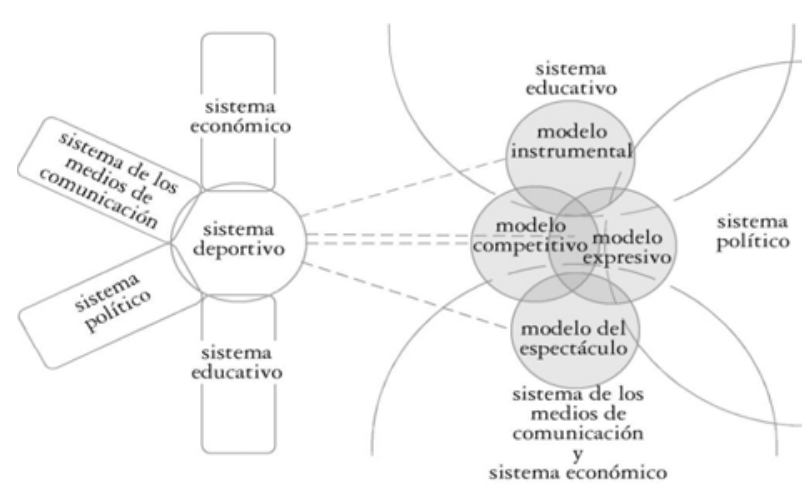

Figura 1. La transformación en el deporte (Puig y Heinemann,1991) 
Haciendo nuestra la clasificación de Heinemann y Puig y, adaptándola a nuestro trabajo, entendemos que los cuatro modelos expuestos por estos autores, es decir, el modelo competitivo, expresivo, instrumental y espectáculo, se podrían agrupar en tres grandes bloques según el nivel de institucionalización de la PAFYD: Institucionalizada (que agruparía los modelos competitivo y espectáculo), Semiinstitucionalizada (que sería casi homologo al expresivo) y ParaInstitucionalizada (que abarcaría, entre otras prácticas de actividad físicodeportiva, la realizada de manera instrumental). Se podría resumir como sigue:

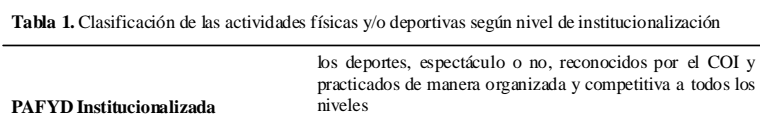

PAFYD Semi-institucionalizad todas aquellas practicas físico-deportivas practicadas de manera lúdico-recreativa, pero que comparten, en esencia, las normas de ejecución de las prácticas de actividad físicodeportivas institucionalizadas

todas aquellas prácticas de actividad fisico-deportivas de nueva creación, apenas institucionalizadas institucionalizadas sin reconocimiento intemacional oficial,

PAFYD Para-Institucionalizada en casos junto a las practicas institucionaliz
margen, e incluso, en otros casos, en contra

Asimismo, y dado que el nivel de institucionalización de las diferentes prácticas de actividad físico-deportiva varía a lo largo de la historia, esta dinámica se podría ilustrar a modo de un átomo donde en el núcleo se concentran las prácticas deportivas más clásicas, las más Institucionalizadas, las de mayor repercusión mediática y, en definitiva, las de mayor reconocimiento social y que no genera ningún tipo de matiz respecto de su carácter de práctica deportiva (ejemplo de ello son todos los deportes, espectáculo o no, reconocidos por el COI y practicados de manera organizada y competitiva a todos los niveles como el baloncesto, futbol, natación,... ). Alrededor del átomo, gravitarían en cada vez mayor distancia, prácticas físico-deportivas de menor nivel de institucionalización que se podrían agrupar en dos grandes grupos: por un lado, las más cercanas al núcleo, es decir las Semiinstitucionalizadas, que agruparían todas aquellas practicas físicodeportivas practicadas de manera lúdico-recreativa, pero que comparten, en esencia, las normas de ejecución de las prácticas de actividad físicodeportivas institucionalizadas; y por otro, las Para-institucionalizadas, todas aquellas prácticas de actividad físico-deportivas denueva creación, apenas institucionalizadas o, institucionalizadas sin reconocimiento internacional oficial, en casos junto a las practicas institucionalizadas, en casos al margen, e incluso, en otros casos, en contra (como el caminar, el parkour, el skate,...). Se podría ilustrar como sigue:

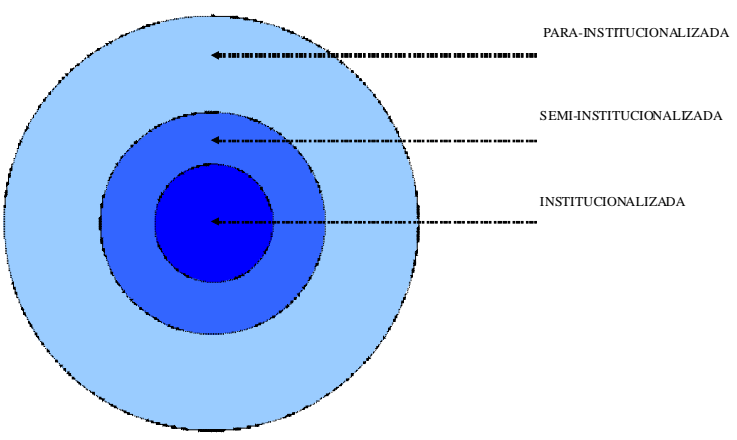

Imagen 2. Metáfora atómica de la dinámica de institucionalización de la Práctica de Actividad Física y Deporte (Fuente: Tesis en preparación de Aldaz Arregui, J.)
Desde esta perspectiva nos proponemos analizar de que manera es cierto el supuesto generalizado de que las personas mayores abandonan la práctica de actividad físico-deportiva, o más bien, transforman el nivel de institucionalización de la misma, con las implicaciones que este hecho conllevaría respecto de la generación de oferta deportiva para con los mayores.

\section{Objetivos}

El propósito de este trabajo es analizar la PAFYD de la población mayor de 65 años de Gipuzkoa ahondando en la dinámica preformativa de la misma que, a nuestro entender, tiene que ver más con una transformación en el nivel de institucionalización de la PAFYD que con un abandono de la misma por causa de la edad.

Para ello, contrarrestamos la perspectiva de los resultados arrojados por el COMPASS y nuestra perspectiva atómica relativa al nivel de institucionalización de la PAFYD.

\section{Material y métodos}

\section{Muestra}

Este trabajo está extraído un estudio mayor cuya muestra total es de 1008 personas de Gipuzkoa de 18 años en adelante. La técnica de muestreo fue aleatoria y estratificada. Con un nivel de confianza del $95 \%$, el error muestral para el total de la muestra se sitúa en $\pm 3,1 \%$. En cuanto a la población mayor de 65 años, objeto de esta comunicación, el nivel de error es del $\pm 5,9 \%$.

La muestra en la que nos centraremos en este trabajo está compuesta por 288 encuestados mayores de 65 años, aunque en ciertos análisis tomamos en cuenta el total de la muestra para realizar comparaciones por rango de edad.

\section{Material}

La recogida de datos fue realizada mediante una encuesta. Uno de los criterios más importantes para recoger los datos es tener una definición clara de lo que se considera PAFYD. En nuestro caso, utilizamos como base la definición consensuada por el proyecto Europeo COMPASS (Coordinated Monitoring of Participation in Sports):

«Entendemos por actividad físico-deportiva toda aquella actividad física que se da mediante la participación organizada o libre, y que tiene como objetivo expresar o mejorar la forma física y mental, divertirse en su tiempo libre, crear nuevas relaciones u obtener resultados competitivos».

\section{Procedimiento}

La recogida de datos fue realizada casa por casa por encuestadores formados siguiendo el procedimiento de rutas aleatorias.

\section{Resultados}

Tal y como hemos tratado de demostrar brevemente en la introducción, la práctica deportiva es difícil de acotar salvo que se entienda desde una perspectiva de un sistema abierto. Esto, se refleja claramente a la hora de analizar los hábitos de práctica de actividad físico deportiva de la población mayor de 65 años.

Si nos atenemos a la metodología COMPASS de una forma estricta, es decir, contemplando las siete tipologías de participación que se derivan de la clasificación de los hábitos de PAFYD según la frecuencia, la pertenencia o no a clubes, y el carácter competitivo de la misma, damos con un esquema analítico de siete perfiles de práctica que se resumen comosigue:

En base a este esquema, observamos que la realidad de los hábitos de PAFYD de la población mayor de 65 años arroja los siguientes resultados: la mayoría de la población guipuzcoana mayor de 65 años, el 79,9\% concretamente, no realiza ningún tipo de PAFYD, 


\begin{tabular}{lccc}
\hline \multicolumn{4}{c}{ Tabla 2: Marco analítico COMPASS } \\
\hline Marco Analítico COMPASS & Frecuencia & Miembro Club & Competitivo \\
\hline Tipo Participacín & $=120$ & Sí & Sí \\
\hline Perfill.Competitivo, organizado, intensivo & $=120$ & No & No \\
Perfil 2.Intensivo & $=120$ & Sí & No \\
& $=120$ & No & No \\
Perfil 3.Regular, Competitivo y/o & $=60 \mathrm{y}<120$ & No & Sí \\
Organizado & $=60 \mathrm{y}<120$ & Sí & No \\
& $=60 \mathrm{y}<120$ & Sí & Sí \\
Perfil 4.Regular, Recreativo & $=60 \mathrm{y}<120$ & No & No \\
Perfil 5.Irregular & $=12 \mathrm{y}<60$ & Sí & Sí \\
& $=12 \mathrm{y}<60$ & Sí & No \\
& $=12 \mathrm{y}<60$ & No & Sí \\
& $=12 \mathrm{y}<60$ & No & No \\
Perfil 6.Ocasional & $=1 \mathrm{y}<12$ & Sí & Sí \\
& $=1 \mathrm{y}<12$ & Sí & No \\
Perfil 7.No Participante & $=1 \mathrm{y}<12$ & No & Sí \\
& $=1 \mathrm{y}<12$ & No & No \\
& ninguno & ninguno & ninguno \\
\hline
\end{tabular}

concentrándose el porcentaje de quienes practican alguna PAFYD en el perfil 2, es decir, el perfil de aquellas personas que realizan PAFYD de manera intensiva.

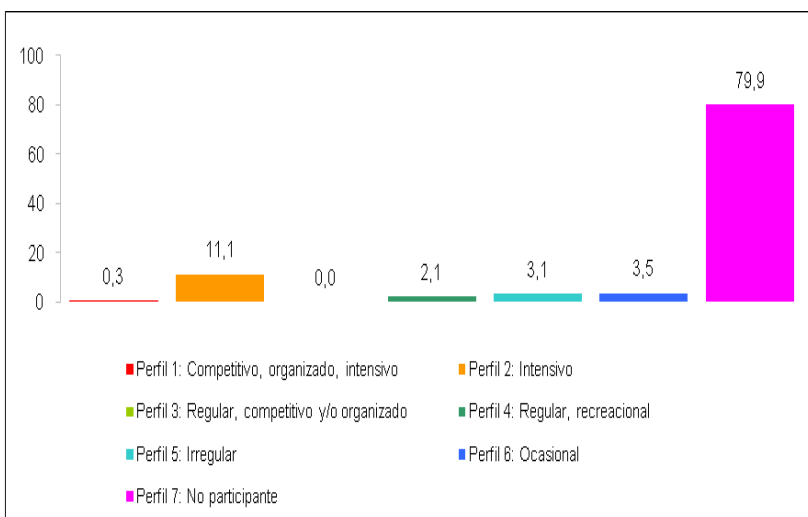

Gráfica 1: \% distribución de la PAFYD según perfiles COMPASS de la población mayor de 65 años

Ahora bien, estos resultados arrojan una realidad parcial que, de quedarnos en los mismos, nos podrían hacer pensar que los mayores de 65 años no realizan PAFYD alguna y que, con la edad, se da un abandono evidente de todo tipo de PAFYD. Estos datos no se corresponden con la realidad que se visualiza en Gipuzkoa, hecho quenos llevó aprofundizar en los datos.

Aplicando un criterio más amplio de PAFYD, y tal y como podemos observar en la gráfica 2, el porcentaje de quienes no realizan PAFYD a partir de los 65 años pasa de ser el 79,9\% aplicando los perfiles COMPASS, al 4,9\% que no realizan ningún tipo de PAFYD ampliando el criterio en cuanto al tipo de práctica física y deporte; en este caso el $75,0 \%$ realiza otra serie de actividades físico-deportivas parainstitucionalizadas, por lo que se observa un mantenimiento en el

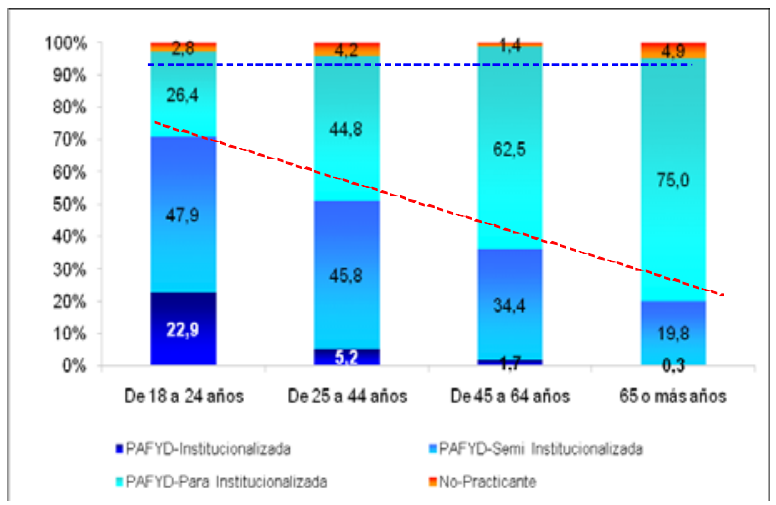

Gráfica 2: \% Practicantes de Actividad Físico-Deportiva según el nivel de institucionalización de la práctica y el intervalo de edad

\begin{tabular}{lr}
\hline \multicolumn{2}{c}{ Tabla 3: \%de las diez modalidades de PAFYD más practicadas por } \\
la población adulta de Gi puzkoa mayor de 65 años \\
\hline 65< (n=288) & $\%$ \\
PAFYD & 92,1 \\
Caminar & 8,0 \\
Natación & 7,6 \\
Cidismo / bicicleta/ mountain bike & 4,5 \\
Aeróbicos, gimnasia de mantenimiento & 3,8 \\
Montañismo/ senderismo & 2,1 \\
Yoga / Tai-chi & 1,4 \\
Caza y pesca & 1,0 \\
Gimnasia deportiva & 1,0 \\
Pelota/ frontón & 0,7 \\
Otros & \\
\hline
\end{tabular}

porcentaje de practicantes, pero no así en el tipo de practica.. Por tanto, la realidad es que la población mayor de 65 años de Gipuzkoa, lejos de dejar de realizar PAFYD alguna, lo que realmente hace es transformarla y adaptarla a sus posibilidades, es decir, deja de realizar PAFYD institucionalizada y semi-institucionalizada, pero aumenta la PAFYD parainstitucionalizada.

De este modo, la dinámica preformativa de los hábitos de PAFYD parece mostrarnos una mayor pertinencia de una hipótesis relativa a la transformación de los mismos, que a un abandono cuanto mayor es la edad aunque, es cierto, que estos datos han de ser complementados con el tipo-modalidad y la frecuencia de la PAFYD parainstitucionalizada que se realiza con el fin de ahondar en esta practica tan extendida entre la población mayor de 65 años de Gipuzkoa.

Si profundizamos en las características de esta PAFYD parainstitucionalizada de la población mayor de 65 años, fijándonos en las 10 modalidades de PAFYD más practicadas, observamos como destaca el caminar sobre el resto de modalidades, con un 92,1\% del total (muy lejos de la segunda modalidad más practicada que es la natación con un $8,0 \%)$.

Asímismo, observamos como laúnica PAFYD que realiza el 69,8\% de los guipuzcoanos mayores de 65 años es caminar, mientras que el 25,3\% realiza otras actividades físicas y deportivas.

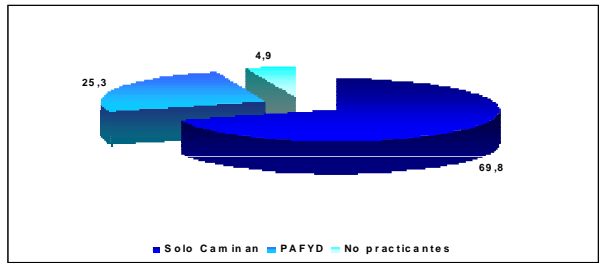

Gráfica 3: Distribución del \% de personas mayores de 65 años que solo caminan, de quienes practican otras actividades físico-deportivas $v$ de no practicantes

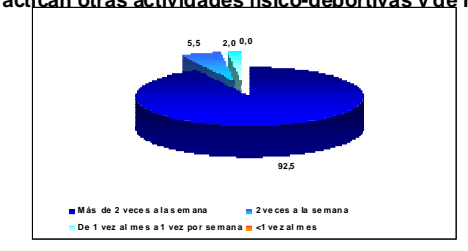

Gráfica 4: Distribución del \% de la frecuencia con la que la población caminante mayor de 65 años camina

El caminar, además de ser la modalidad que más personas mayores de 65 años practican en Gipuzkoa, cabe decir que no nos encontramos ante una práctica anecdótica, ni mucho menos, dado que, tal y como podemos observar a continuación (gráfica 4), las personas que como PAFYD únicamente caminan (el 92,5\%), lo hacen mayoritariamente con una frecuencia de tres o más veces a la semana.

\section{Discusión y conclusiones}

Tal y como hemos tratado de mostrar hasta aquí, los hábitos de PAFYD de la población guipuzcoana mayor de 65 años nos muestran como la población adulta, con el paso de los años, va tendiendo hacia 
una PAFYD menos institucionalizada, lo cual no quiere decir que se dé un abandono sino que nos hace pensar que la dinámica preformativa de los hábitos de PAFYD tiene que ver más con una transformación que con el abandono de los mismos.

Este hecho nos abre un espacio para el estudio relacionado con la necesidad de ahondar en los mecanismos a través de los cuales se institucionalizan las actividades físico-deportivas y la falta de ajuste entre el modelo de institucionalización de la oferta de PAFYD institucionalizada actual y la demanda, de facto o no, de la población guipuzcoana mayor de 65 años.

Es por ello que la metáfora atómica de los hábitos de PAFYD nos hace pensar en que si bien es cierto que las prácticas más institucionalizadas son las practicadas por la población más joven, se habría de ampliar el núcleo institucionalizado adaptándolo a los potenciales para la PAFYD que presenta la población mayor de 65 años rompiendo con el modelo actual que reproduce un modelo organizativo de PAFYD que excluye progresivamente a la población cuanto mayor desde un falso axioma de que el proceso vital de envejecer excluye de toda PAFYD. Quizá haya que plantearse seriamente el hecho de si no es más el modelo de institucionalización del sistema deportivo actual el que excluye a las personas mayores que el propio proceso biológico de envejecer, y la responsabilidad de las instituciones públicas en el cambio de dicho modelo.

A nuestro entender, tanto las metodologías enfocadas a la medición de los hábitos de PAFYD, como las políticas implicadas en la promoción de los mismos, si bien han vivido grandes avances en los últimos tiempos, atendiendo a la mayor esperanza de vida de civilizaciones como la nuestra, debieran de atenderse de forma seria aspectos tales como el tipo de PAFYD que atrae y es saludable para los y las mayores de 65 años.

Para concluir diremos que consideramos necesaria la reformulación de los modelos de institucionalización predominantes así como las políticas relacionadas con los hábitos de PAFYD de la población adulta, en general, y de los mayores de 65 años, en concreto.

\section{Bibliografía}

AA.VV. (2007). Libro de Actas del II. Congreso Internacional de Actividad Físico Deportiva para Mayores. Málaga: Conserjería de Turismo, Comercio y Deporte / Instituto Andaluz del Deporte.

ARRIBAS, S. (2004). La Práctica de la Actividad Física y el Deporte (PAFYD) en escolares de 15-18 años de Gipuzkoa: creencias sobre su utilidad y relación con la orientación motivacional, diversión y satisfacción. Leioa: Servicio editorial de la Universidad del País Vasco.

ARRIBAS, S. y ALDAZ, J. (Eds.) (2008). Hábitos, estilos de vida y salud relacionados con la actividad física y el deporte. Bilbao: UPV/EHU.

ARRUZA, J.A. y ARRIBAS, S. (2001). Estudio sobre los Hábitos Deportivos de la población de Guipúzcoa. Donostia-San Sebastián: Guipúzcoako Foru Aldundia. Kirolarte.

ARRUZA, J, ARRIBAS,S. GIL DE MONTES, L (2007). Hábitos de Práctica Deportiva: Análisis de las relaciones entre el sedentarismo y la salud psico-física. I. S. C. F. MANUEL FAJARDO. AFIDE. II Convencion Internacional de Ciencias de la Actividad Fisica y el Deporte. La Habana

BIDDLE, S.J.H., FOX, K.R., \& BOUTCHER, S.H. (2000). Physical activity and psychological well-being. London: Routledge.

BIDDLE, S.J.H. \& MUTRIE, N.(2001). Psychology of physical activity: Determinants, well-being and interventions. London: Routledge.

CAMPILLO, J.E. (2004). El mono obeso. La evolución humana y las enfermedades de la opulencia: diabetes, hipertensión, arteriosclerosis. Barcelona: Ed. Critica.

CANTERA, M.A., y DEVÍS-DEVÍS, J. (2000) «Actividad física y salud», en Apunts, 67 (pp. 54-62)

CERVELLÓ, E. (1996) La motivación y el abandono deportivo desde la perspectiva de las metas de logro. Tesis doctoral. Universidad de Valencia.
DFG Kirol Zuzendaritza Nagusia. (2008) «Diez ejes de política deportiva para el período 2008-2011»

DUBBERT, P.M., KING A.C., MARCUS, B.H., \& SALLIS, J.F. (2004). Promotion of physical activity through the life span. In Boll, T.J., Raczynski, J.M., \& Leviton, L.C. (2004). Handbook of clinical and health psychology (pp. 147-180). Washington DC: American Psychological Association.

GARCIA FERRANDO, M. (1996) «Cambios en los hábitos deportivos de los españoles», en Temas para el debate, 23 (pp. 43-46)

GARCIA FERRANDO, M. y MESTRE SANCHO, J.A. (2002) Los hábitos deportivos de la población de Valencia. Valencia: Fundación Deportiva Municipal

GARCIA FERRANDO, M. (2006) Posmodernidad y Deporte: entre la individualización y la masificación. Madrid: CIS.

GUTIERREZ, M. (2000) «Actividad física, Estilos de vida y Calidad de vida», en Revista de Educación Física, 77, (pp. 5-14)

MUSSINO, A. (2002). COMPASS 2002. Progress Raport, Roma.

PORRAS SÁNCHEZ,M; DÍAZ GARCÍA,J. (2007). Hábitos y actitudes de los sevillanos mayores ante el Deporte. Observatorio del deporte de Sevilla. Sevilla

PUIG, N. y HEINEMANN, K. (1991). «El deporte en la perspectiva del año 2000», en Papers, 38, 123-141.

ROMERO, S, et. Col (2007). Hábitos de práctica de actividad físicodeportiva yestilos de vida en la población adulta de Sevilla. I. S.C. F. MANUEL FAJARDO. Ponencia. AFIDE. II Convencion Internacional de Ciencias de la Actividad Fisica y el Deporte. La Habana

STATHI, A., FOX, D.R., \& MCKENNA, J. (2002). Physical activity and dimensions of subjective well-being in older adults. Journal of Aging and Physical Activity, 10, 76-92.

STAHL, T., RÜTTEN, A., NUTBEAM, A., BAUMAN, L., ABEL, G., LÜSCHEN, G., RODIRGUEZ, J.A., VAN DER ZEE, J. (2000). The importance of the social environment for physically active lifestyle - results from an international study. Social Science and Medicine, 52, 1-10.

WANKEL, L.M. (1993) «The importance of enjoyment to adherence and psychological benefits from physical activity», en International Journal of Sport Psychology, 24 (pp.151-169)

Trabajo realizado gracias a la ayuda de los Fondos FEDER. Ministerio de Educación y Ciencia (DEP2006-56054-C03-01/ACTI), y la colaboración del Servicio de Deportes Diputación Foral de Gipuzkoa y www.ehu.es/cinvidere/.

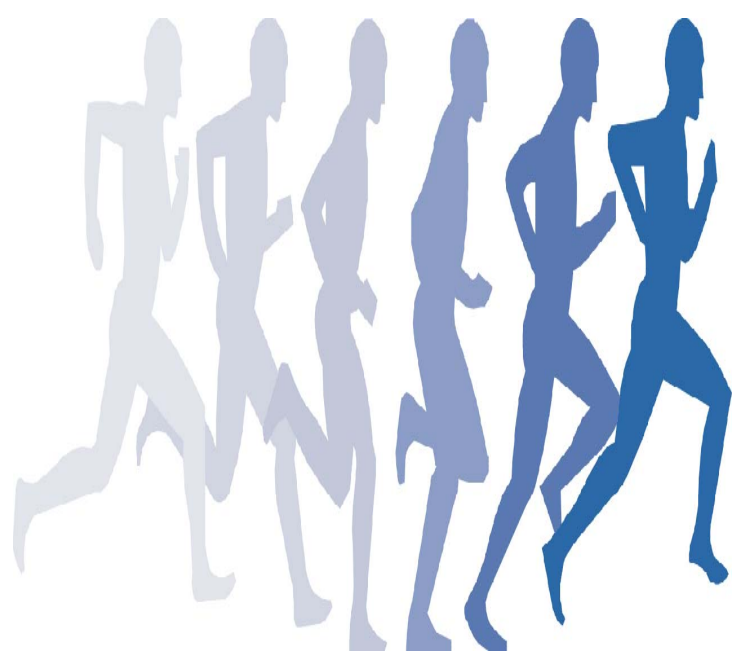

